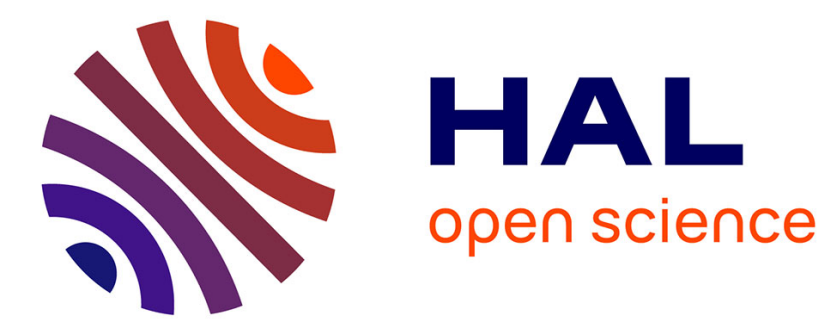

\title{
Habiter : ce que le périurbain nous apprend
}

Rodolphe Dodier

\section{To cite this version:}

Rodolphe Dodier. Habiter: ce que le périurbain nous apprend. Travaux de l'Institut de Géographie de Reims, 2006, 29-30 (115-118), pp.31-44. halshs-00189925

\section{HAL Id: halshs-00189925 https://shs.hal.science/halshs-00189925}

Submitted on 22 Nov 2007

HAL is a multi-disciplinary open access archive for the deposit and dissemination of scientific research documents, whether they are published or not. The documents may come from teaching and research institutions in France or abroad, or from public or private research centers.
L'archive ouverte pluridisciplinaire HAL, est destinée au dépôt et à la diffusion de documents scientifiques de niveau recherche, publiés ou non, émanant des établissements d'enseignement et de recherche français ou étrangers, des laboratoires publics ou privés. 


\section{Rodolphe Dodier, Université du Maine, GREGUM / ESO, UMR CNRS 6590}

\section{Habiter : ce que le périurbain nous apprend}

\section{Résumé :}

Le périurbain est actuellement un espace-clef des dynamiques socio-spatiales, avec une oscillation entre émergence d'un mode de vie spécifique et continuité de l'osmose avec la ville. Contrairement aux représentations dominantes, cet espace est composé de segments immobiliers différenciés (au-delà de l'apparente banalité des lotissements) avec une hétérogénéité importante des statuts socio-économiques et des structures démographiques plus homogènes (domination des familles). Le rapport à l'espace des ménages périurbains est cohérent avec sa composition sociale, avec un fort rapport au logement caractéristique des familles. Cependant l'analyse de l'habiter périurbain montre la variété des identités spatiales. Le rapport au village périurbain oscille entre fort sentiment identitaire et utilisation comme simple commune-dortoir, le rapport à la ville variant entre attraction et répulsion. Cela permet aussi de montrer l'individualisation croissante des pratiques spatiales et la difficulté à traduire l'éclatement des espaces de vie et leur nature réticulaire.

Mots-clefs :

Habiter, espace vécu, périurbain, spatialité

\section{Abstract :}

At present, peri-urban space is an important place to examine social and spatial dynamics, with an oscillation between the emergence of a specific way of life and osmosis with the town. Contrary to prevailing representations, this space is composed of different types of housing (not only ordinary housing estates), with an important heterogeneity of economic positions and a more homogeneous demographic structure (families predominate). The relationships between household and space are coherent with the social composition of periurban space, and show that families have a strong relationship with the place they inhabit. However, the analysis of dwellings in peri-urban space shows the diversity of spatial identities. The relationship with the peri-urban space oscillates between strong identity as a member of a living community or indiferrent residence in a mere dormitory town, as for the relationship with the town, it fluctuates between attraction and repulsion. With this analysis we can show the increased individualization of spatial practices and the difficulty to express the dispersion of lived space and its reticular nature.

Key-words :

Dwelling, lived space, peri-urban space, spatiality

Le but de cet article est d'utiliser un ensemble de recherches de terrain menées dans l'espace périurbain afin de dégager quelques apports pour la problématique de l'habiter.

L'intérêt du périurbain est lié aux représentations qui y sont associées et aux débats qui traversent actuellement les recherches sur la ville éclatée : émergence d'une véritable société autonome ou continuité d'un fonctionnement social et économique en osmose avec la ville? Dans un certain nombre de travaux, l'urbanité périurbaine est foncièrement suspecte. Repli sur soi, fuite de la ville, manque de sociabilité, choix résidentiel sous contrainte (Bonvalet et Dureau 2000) générateur de frustration, éloignement de la ville générant une 
mobilité intense mal vécue (Baudelle et al. 2004), contradiction entre retour à la nature et pollution individuelle liée au tout automobile (Sajous 2004), réactions de rejet des équipements collectifs avec des phénomènes de type NYMBY, fort vote Front National (Grésillon 1998), etc., les lieux communs abondent, parfois sur une base objective mais aussi à partir de représentations négatives de la part de chercheurs fondamentalement urbains débouchant sur une forme de stigmatisation de ces espaces face à la montée du concept de développement durable (Brück et al. 2001). Une réaction récente (Estèbe 2004) vient de faire une réponse intéressante à un certain nombre de ces représentations, mais plus sur le mode de l'incantation que sur des arguments réellement objectifs, excepté sur la question de l'intercommunalité.

En fait, au-delà des représentations trop simplistes, l'immersion dans le périurbain, avec ses modes de vie éclatés, sa véritable multi-appartenance territoriale frisant la schizophrénie, en particulier dans le périurbain lointain, le tiraillement incessant entre une communauté villageoise idéalisée mais parfois désenchantée et un espace de vie principal, la ville, pour lequel les ménages oscillent entre attraction et répulsion, permet de mettre en évidence à la fois l'hétérogénéité sociale des habitants et l'existence d'identités spatiales complexes.

Cette analyse des spatialités périurbaines permettra de proposer quelques pistes de réflexion pour la problématique de l'habiter. Selon la définition qui est donnée de l'habiter (large et peu ou prou équivalente à spatialité ou au contraire centrée sur le logement), l'accent sera mis soit sur l'individualisation des identités spatiales, soit sur la difficulté à transcrire l'éclatement des espaces de vie et leurs structures réticulaires.

Le terrain utilisé est l'espace périurbain d'une ville intermédiaire, Le Mans, qui n'a pas de spécificité majeure : la structure d'activité est proche de la moyenne française après avoir été assez industrielle, la structure de qualification est marquée par une légère sousreprésentation des cadres surtout comparée aux métropoles régionales et a fortiori à Paris, les prix du foncier et de l'immobilier restent assez faibles bien qu'en augmentation, les pathologies urbaines (délinquance en particulier) ne sont pas très significatives, et le vivreensemble a encore une signification. (Bertrand et al 2000). Tous ces éléments vont jouer sur la composition sociale du périurbain et sur la nature de l'articulation entre la ville et son espace périurbain. Le contexte non métropolitain explique sans doute une moindre propension à l'entre-soi, ce qui est caractéristique des villes intermédiaires et moyennes. Enfin, l'image des $24 \mathrm{~h}$ ne doit pas faire illusion, car si la ville est un haut lieu du tourisme international une petite semaine par an, ce n'est qu'une simple parenthèse. Le contexte est donc ordinaire, ce qui permet d'imaginer une transposition à un grand nombre de villes intermédiaires et moyennes.

Les lieux d'observation associent communes périurbaines lointaines et communes plus proches de la ville, avec des aspects comparatifs. Une première phase a consisté à s'interroger sur les flux domicile-travail, à caractériser leur composition sociale (traitements quantitatifs), démontrant le tri social progressif entre les différentes couronnes périurbaines, les ménages populaires étant «rejetés » de plus en plus loin de la ville-centre alors que les catégories moyennes ou supérieures investissent fortement la première couronne.

La seconde phase, à partir d'entretiens permettant d'être plus interprétatif que descriptif, nous a conduit à nous intéresser aux stratégies résidentielles des ménages (Dodier 2004), en essayant de réintroduire la dimension spatiale souvent occultée, en particulier en insistant sur les arbitrages entre des proximités relatives à différents types de lieux dans une situation de choix limité pour les ménages et de transparence imparfaite du marché immobilier. 35 entretiens ont été menés jusqu'à présent : certains sont relativement classiques, du type semi-directif avec un interlocuteur unique ou éventuellement les deux membres du ménage, en essayant de pratiquer la méthode du double entretien (Hoyaux 2003) ou à défaut 
en confrontant les interprétations in situ à l'appréciation des personnes concernées. Une dizaine de ces entretiens sont des entretiens collectifs sous forme participative, soit à l'occasion d'une réunion formelle (par exemple des comités de direction ou des bureaux d'associations), soit de façon plus informelle (repas d'amis ou de collègues, notamment pour les cadres d'une entreprise d'équipements automobiles), ce qui permet d'approfondir les interprétations par leur mise en débat au sein du groupe constitué.

Dans le cadre de cet article, le but n'est pas de retranscrire ces entretiens ni de proposer une interprétation de certains d'entre-eux, mais de faire une lecture synthétique des pratiques spatiales des ménages résidant dans le périurbain et de leurs identités spatiales.

\section{Diversité des segments de l'immobilier et diversité sociale}

Le premier apport concerne l'hétérogénéité du périurbain, élément sur lequel insistent peu les travaux quantitatifs sur le périurbain (Guilly et Noyé 2004). Certes, l'éventail des situations sociales et des modes de vie est peut être un peu plus faible qu'en milieu urbain, mais s'il existe une relative unité démographique, la diversité des situations sociales et des rapports à l'espace ressort de façon forte.

Ainsi, sous l'apparente banalité des lotissements, se cache en fait à la fois une importante hétérogénéité du bâti et une grande diversité de la façon d'occuper son logement et de la façon de le mettre en scène sur le plan social, ce qui correspond à une première acception d'habiter. Au niveau du bâti, l'image du lotissement, parce qu'elle est très largement dominante, occulte largement les autres formes spatiales alors que trois formes principales coexistent dans le périurbain, notamment dans le périurbain lointain, associées à une forme secondaire.

La forme dominante est le lotissement, mais entre les lotissements avec de grandes parcelles de près de $2000 \mathrm{~m}^{2}$ situés en première couronne, avec une évidente sélectivité sociale (forte proportion de cadres supérieurs), et les lotissements avec des tailles moyennes de parcelles de l'ordre de $800 \mathrm{~m}^{2}$ (voire moins), plus nombreux dans le périurbain lointain et qui accueillent plutôt des catégories populaires solvables, il existe tout une graduation de situations sociales dont le lien avec le coût du foncier (et donc la distance à la ville) et l'ancienneté du lotissement est évident. Dans ces lotissements, le mode de vie des habitants est mis en évidence par les variations possibles à partir du modèle standard, avec des choix architecturaux plus ou moins marqués, avec une plus ou moins grande capacité à la transformation, marquant parfois l'appartenance aux catégories moyennes supérieures (le petit chalet en bois pour abriter la tondeuse et les outils de jardin), signifiant d'autres fois l'appartenance à des catégories plus populaires (on fabrique son appentis avec des matériaux de récupération). A l'intérieur du logement, le mobilier et la fonction assignée à chaque pièce sont également des marqueurs sociaux, entre le modèle émergent de la grande pièce à vivre caractéristique des classes moyennes supérieures et la pérennité de la cuisine comme pièce de la convivialité populaire, avec des modèles intermédiaires fondés sur des utilisations différenciés de la salle à manger, du séjour ou du salon. Le mode de mise en valeur du terrain, parce qu'il donne aussi à voir aux voisins, est également un marqueur déterminant de la position sociale. Dans la première couronne, le jardin d'ornement domine, avec une profusion de fleurs pendant le printemps et l'été, une mixité entre arbres fruitiers et d'ornement, avec même de plus en plus une illumination lors des fêtes de Noël et, éventuellement, avec une petite place pour un potager qui est plus de l'ordre du folklore que de l'utilité sociale. Par contre, plus dans le périurbain lointain, la place du potager redevient importante, repassant en façade pour donner à voir le savoir-faire et avec une fonction économique qui reprend un véritable sens, notamment dans les cas de surendettement. 
La seconde forme est constituée par les fermettes rénovées, auxquelles on peut désormais adjoindre les pavillons isolés, de moins en moins nombreux à être construits et qui pour une grande part datent des années 1970. Dans ce type de bâti, la sélectivité sociale est beaucoup plus importante. Deux populations vont en fait résider dans ces fermettes : d'une part les «bricoleurs », spécialistes de la remise en état de bâtiments délabrés, et qui pour certains en sont à leur deuxième voir leur troisième localisation résidentielle dans le périurbain, ce qui s'est traduit par des plus-values non négligeables; d'autre part, des catégories sociales aisées, en particulier dans la première couronne où ce type de bâti a pris une valeur importante en raison des aménités environnementales qui lui sont associées tout en restant à une distance raisonnable de la ville-centre. Dans ce type de maisons se retrouvent les mêmes modes distinctifs qu'entre pavillons des lotissements.

La troisième forme est constituée par les maisons de bourgs, généralement de taille très modestes, qui semblent vraiment méconnues dans la littérature existante, sans doute parce qu'autour des grandes villes ce type de bâti est devenu minoritaire. Dans ce type de bâti, l'hétérogénéité sociale est beaucoup plus importante. D'abord, la proportion de familles est beaucoup plus faible que dans le reste du périurbain, avec plus de personnes âgées, souvent d'origine rurale et qui vivent parfois dans des situations financières pour le moins difficiles, mais aussi plus de jeunes couples ou des célibataires, d'origine urbaine, qui peinent de plus en plus à trouver un appartement dans le logement social ou dans le secteur privé dans la proche ville (forte proportion de RMI). Ces personnes se retrouvent à faire des navettes d'une vingtaine de kilomètres, générant des coûts de transports importants vu leurs revenus (forte proportion de travail précaire). Il existe donc ici une strate sociale peu favorisée, certes moins visible que dans les quartiers d'habitat social et avec sans doute moins de problèmes comportementaux (forte régulation par les communes).

A cette forme spécifique, s'ajoute de plus en plus du logement social, voire très social, construit récemment par exemple dans le cadre des lois sur l'exclusion et des PLH, et qui accueille des populations très modestes, avec des situations économiquement difficiles et des situations familiales complexes.

Au bout du compte, l'éventail des situations sociales se retrouve aussi varié qu'en milieu urbain, même si les extrêmes sont numériquement moins nombreux (populations avec des difficultés d'une part, populations très aisées d'autre part), avec une surreprésentation surtout marquée en termes de types de ménages, avec une très large majorité de familles avec enfants. Plus qu'une spécificité de statut socio-économique, le périurbain est donc marqué par une spécificité liée au cycle de vie des ménages, qui se traduit dans les formes d'occupation du logement.

L'homogénéité du périurbain résulte donc des structures démographiques et de la position dans le cycle de vie plus que d'un positionnement socio-économique. Cela permet de mettre en évidence la diversité sociale du périurbain et de s'éloigner des représentations trop rigides du type «univers pour les classes moyennes » (Jaillet 2004) ou «place du peuple » (Estèbe 2004).

\section{Les pratiques spatiales dans le périurbain}

Le deuxième ensemble de résultats provient de l'analyse des pratiques spatiales des ménages habitant le périurbain et en particulier de l'exploration des rapports successifs au logement, au village périurbain (pour lequel il faut faire un parallèle avec le quartier urbain) et à la ville dans sa globalité. A travers ces pratiques spatiales, il sera ainsi possible de revenir sur la question de la fuite de la ville et du repli sur soi supposé de ces populations périurbaines, pouvant traduire l'émergence d'un habiter périurbain spécifique. 
Dans un premier temps, il paraît important de rappeler l'extrême diversité des pratiques spatiales. De ce point de vue, les individus apparaissent vraiment comme des acteurs, effectuant des choix et les assumant. Si quelques tendances peuvent être observées selon les catégories sociales, ce ne sont vraiment que des tendances, la diversité des rapports à l'espace étant très marquée au sein d'une même catégorie sociale. Ainsi, deux exemples, proches à la fois dans leur situation spatiale (la même commune du périurbain lointain du nord du Mans) et dans la situation sociale des individus (catégories aisées, un médecin et un ingénieur) montrent bien cette diversité. Pour M. (le médecin), la pratique du logement est certes intense (réceptions fréquentes), mais la connaissance de l'ensemble du canton où il exerce est également très importante, avec une perception très fine à la fois des lieux et des populations et une implication locale forte dans plusieurs associations (culturelles notamment). Il n'hésite pas non plus à fréquenter la ville de façon très assidue, plusieurs soirées par semaines, pour des activités culturelles (dont-il est très friand), pour des relations sociales, et de temps en temps pour des raisons professionnelles avec une implication associative dans ce cadre. Cette personne a donc des pratiques spatiales impliquées à la fois de son logement, de l'espace périurbain environnant son lieu de résidence et de la ville, sans se limiter au Mans d'ailleurs. A contrario, pour J., la ville n'est « que » le lieu de travail, avec juste éventuellement un arrêt pour faire quelques courses dans l'hypermarché d'entrée de ville qui est sur son trajet, et il n'y développe pas de relations sociales (sauf quelques relations professionnelles « contraintes »). Dans sa commune, il n'est pas «intégré » selon ses propres termes, n'entretient que des relations de courtoisie avec ses voisins (physiquement assez éloignés d'ailleurs) et ne fréquente pour ainsi dire que la boulangerie. L'essentiel de son temps hors travail est en fait consacré à l'entretien de sa maison et de son grand terrain et à la lecture avec achat des livres par correspondance. Sa vie est donc très centrée sur son logement, sans qu'il y reçoive beaucoup d'ailleurs (essentiellement quelques membres de la famille de sa femme). J. a donc des pratiques spatiales peu impliquées et très centrées sur le logement et revendique ce repli sur le domicile.

Plus généralement, même si les effectifs analysés sont réduits, les profils de repli sur le logement sont assez rares et le fait de personnes âgées d'origine rurale plutôt que de ménages dans lesquels au moins un des actifs travaille dans la ville ou sa proche périphérie. Le rapport au logement est par contre très souvent assez important, manifestement plus que dans les quartiers centraux des grandes villes (Authier 2001). Il s'agit cependant d'un effet de structure de la population, lié à la nette surreprésentation des familles. Ainsi, le rapport élevé au logement s'accompagne d'une forte activité de transformation, y compris dans le neuf, avec des logements qui évoluent au fur et à mesure des besoins du ménage (pièce nouvelle lors de l'arrivée d'un troisième enfant par exemple). La transformation est aussi fonction de la capacité financière du ménage qui peut avoir réservé un espace pour des agrandissements futurs (le fameux grenier aménageable). La pratique du jardinage ou de façon plus générale l'entretien du terrain complète ce fort investissement dans le logement. Les pratiques familiales (le repas du soir pris en commun, un certain nombre d'activités réunissant enfants et parents, même si on est parfois plus dans l'idéalisation de la vie de famille que dans sa pratique concrète), la réception d'amis ou de la famille proche, complètent ce fort rapport au logement.

Toutefois, avant d'interpréter ce fort rapport au logement comme un repli sur soi, il convient de s'intéresser aux autres dimensions des pratiques spatiales. Interpréter un fort rapport au logement comme un repli sur la cellule familiale ne peut se faire que s'il s'accompagne d'une faible implication aux autres échelons. 


\section{Un rapport au village périurbain entre idéal communautaire et détachement}

Au niveau du rapport à la commune périurbaine et éventuellement à ses environs, la diversité est beaucoup plus importante. Rappelons qu'en milieu urbain, une personne sur deux seulement à un rapport moyen ou élevé au quartier (Authier 2001). Or, pour l'espace périurbain, c'est la commune périurbaine qui fait office de quartier, même si, comme en ville, certaines personnes ont une vision du quartier limitée à la rue ou au lotissement ou au contraire élargissent l'espace du quotidien à quelques communes contiguës.

Parmi les entretiens effectués, S., technicien, ressort très nettement comme l'archétype de la personne très investie dans la vie de l'échelon local. Président de deux associations (une à objet de sport-loisir, l'autre à but culturel), membre de trois autres associations, sollicité pour rejoindre le conseil municipal, il anime et participe à la plupart des manifestations organisées dans sa commune du périurbain du sud-ouest du Mans. Il a développé des relations amicales avec de nombreux habitants de sa commune, en reçoit plusieurs fois par semaine dans son logement, part même en vacances avec certains d'entre eux. S. revendique d'être un véritable «citoyen de sa commune ». En fait, les pratiques spatiales et sociales dans les communes périurbaines sont généralement moins intenses que dans ce cas spécifique, mais associent généralement des usages peu impliquants (quelques achats dans les commerces locaux, fréquentation de l'école et activités du mercredi pour les enfants, activités dans un cadre associatif mais dans une optique de consommation pour les parents) et des relations de sociabilité qui ont souvent un sens beaucoup plus fort. Dans une des communes de notre échantillon, la proportion de personnes participant aux activités culturelles ou sportives, aux repas des anciens ou au goûter des écoles, est particulièrement importante. La participation aux nombreuses fêtes est également assidue (250 personnes à chaque fois pour une population de moins de 1000 habitants). Toutefois, il ne faut pas non plus idéaliser cette participation, une frange de la population (40\% ?) ne semblant jamais participer aux animations locales.

Etant donné la taille de notre échantillon, il est difficile de quantifier ce rapport à la commune de résidence, mais il semble y avoir un rapport au local un peu plus fort que dans d'autres espaces urbains, avec une importante diversité selon les personnes. Certains ménages résident vraiment dans une commune-dortoir, simple support d'accueil de leur logement, avec quelques aménités environnementales surtout recherchées pour la santé des enfants et des services souvent considérés comme insuffisants. D'autres, souvent par volonté manifeste lors de leur choix résidentiel ( on est venu chercher autre chose que l'anonymat de la ville»), s'investissent plus dans les relations sociales avec les voisins. Dans ce cas, l'évolution à long terme peut parfois être source de distorsion entre la vie sociale rêvée, avec une certaine idéalisation de la convivialité rurale, et la réalité du fort contrôle social (qui était aussi historiquement l'apanage des relations sociales en milieu rural) ou le décalage entre leurs attentes et celle de leurs voisins. A contrario, certaines personnes, arrivées dans le périurbain par les contraintes financières et sans véritable volonté d'intégration, racontent comment, par l'école dans un premier temps et par les animations culturelles dans un second temps, elles ont été amenées à avoir de nouvelles relations sociales dans la commune de résidence. $\mathrm{Au}$ final, les relations à l'espace périurbain semblent globalement un peu plus fortes que les relations au quartier en milieu urbain, infirmant en grande partie les accusations de repli sur soi qui fleurissent actuellement dans la littérature scientifique.

\section{Un rapport à la ville entre attraction et répulsion}

Pour les rapports à la ville, les discours sont nettement plus difficiles à interpréter, et il peut être judicieux de s'intéresser d'abord aux pratiques spatiales. Ainsi, la ville reste le lieu de travail de la très grande majorité des périurbains actifs, plus de $85 \%$ en deuxième 
couronne (le chiffre est un peu plus faible dans la première couronne suite à l'amorce du desserrement des activités), pour ensuite diminuer régulièrement avec la distance au centre. La composition sociale de ces flux change également, avec une proportion croissante d'ouvriers vers l'extérieur. Le double effet de la distance et du statut social se combine pour expliquer un lien avec la ville de plus en plus distendu avec l'éloignement au centre, bien que de nombreuses configurations différentes s'observent dans chaque couronne.

Plutôt dans la première couronne et plutôt parmi les catégories sociales moyennes ou supérieures, se trouvent des individus ayant toujours un fort rapport à l'urbain. La consommation se fait prioritairement en ville, à la fois quotidienne sur le trajet au lieu de travail, hebdomadaire dans un hypermarché de la périphérie, mais aussi plus rare dans les magasins spécialisés du centre où on aime encore à faire du shopping. Les activités culturelles, notamment le cinéma, les activités sportives ou de loisirs, en particulier celles des enfants le mercredi mais aussi les activités un peu spécifiques des parents, tout cela a lieu aussi dans la ville. Enfin, pour un grand nombre de ménages, les relations de sociabilité n'ont pas fondamentalement changé du fait de leur déménagement dans le périurbain. Au contraire, dans un certain nombre de cas, le nouveau logement est devenu le lieu de ralliement du groupe d'amis («on a plus de place et puis avec le barbecue c'est sympa »). La ville est donc le lieu de relations sociales souvent construites anciennement (les anciens voisins, les amis d'enfance, les collègues de travail ou les relations dans le cadre associatif), qui structurent profondément l'identité sociale de l'individu ou du ménage. D'ailleurs, la fin de la vie active ou plus encore le départ des enfants se traduisent pour ces ménages par une pratique plus forte de la ville et de ses sociabilités, et parfois par un retour résidentiel au centre. Dans ce cas, l'urbanité reste forte et il n'est pas question de l'émergence d'un mode relationnel spécifique ou d'une autonomisation du périurbain.

Plus loin en périphérie du bassin d'emploi, éloignement et statut social plus modeste expliquent effectivement une plus grande prudence vis-à-vis de la ville, pas forcément un rejet mais une indifférence à ce qui s'y passe. Mais un grand nombre de ces ménages ont-ils jamais été urbains ? Contraintes par les difficultés de transport de l'époque de leur arrivée en ville, un certain nombre de personnes d'origine rurale et devenues urbaines (et plus généralement ouvrières) dans les années 1950 et 1960, ainsi que leurs enfants eux aussi devenus ouvriers, ne sont pas mécontentes de retrouver un espace au moins rural dans sa morphologie. Dans ce cas, la ville n'a jamais été complètement investie, elle a pu toujours apparaître comme un organisme étrange qu'on est soulagé de quitter, même au prix de coûts de transports élevés. La fréquentation de la ville se réduit alors de façon forte, n'étant plus que le support du lieu de travail des parents, du lieu d'étude éventuel des enfants, doublés de la fréquentation de l'hypermarché le samedi. Dans ce périurbain lointain, là où on vote plus Front National (en grande partie par effet de structure cependant), les réactions de rejet de la ville, mais aussi le sentiment, pas complètement subjectif quand on voit le manque d'équipements et de services, d'être rejeté par la société dominante, se généralisent d'autant plus facilement que tout cela se greffe sur des situations familiales parfois difficiles (surendettement, précarité du travail ouvrier, mal de vivre des adolescents, etc.).

Entre ces deux extrêmes, un grand nombre de ménages oscillent entre dédain et attraction pour la ville. Ils justifient leur choix sous contrainte par les arguments habituels (opportunité foncière, recherche de la proximité de la nature pour les enfants, rejet de la vie urbaine), mais en même temps reconnaissent l'utilisation des avantages de la ville et laissent entrevoir un certain attachement. Concrètement, leurs pratiques spatiales dans la ville restent importantes, même s'ils évitent le centre-ville pour des raisons pratiques. Ils continuent d'avoir des relations de sociabilité sur l'ensemble de la ville, même si elles se distendent progressivement. S'ils reçoivent ou se déplacent moins souvent, c'est aussi à cause des enfants, on se reçoit plus entre familles, c'est la fin des sorties dans le cadre du groupe d'amis. 
Le bilan des rapports à la ville est donc pour le moins contrasté. Certes, globalement la fréquentation impliquée de la ville semble moins courante que pour les habitants des quartiers centraux, mais c'est aussi un effet de structure, lié à la nette surreprésentation des familles qui ont des rapports privilégiés au logement. Il est d'ailleurs probable que ce rapport à la ville est aussi important que celui des ménages avec enfants résidant dans les quartiers d'habitat social situés à la périphérie des grandes villes. Traduire l'oscillation entre rejet et fascination pour la ville des ménages du périurbain comme une fuite de la ville ou un repli sur soi semble donc être un raccourci un peu rapide, au moins dans le contexte de la ville intermédiaire.

\section{Individus ou ménages ?}

De cette immersion dans le périurbain, ressortent quelques enseignements qui peuvent donner des pistes pour la problématique de l'habiter.

Premièrement, le raisonnement au seul échelon de l'individu semble en partie voué à l'échec. S'agissant de familles, généralement avec plusieurs enfants, l'identité territoriale de chaque individu évolue aussi au rythme des besoins sociaux du ménage et, spécifiquement, des relations sociales des différents membres du ménage. Si, lorsque les enfants sont petits, la territorialité étroite de la commune périurbaine, éventuellement élargie à quelques communes contiguës, complète parfaitement, pour les parents, une seconde identité territoriale liée au travail à la consommation et aux sociabilités urbaines, lorsque les enfants grandissent, c'est cette identification étroite qui est source de conflits familiaux, voire de reconstruction d'autres identités à la fois familiales et territoriales. Certains entretiens montrent très bien les conflits internes aux ménages et les arbitrages qui sont finalement effectués. L'un d'entre eux fut brutalement interrompu par l'intervention d'un adolescent pour qui « ouais, ici c'est la zone, je peux même pas sortir le soir parce que vous voulez pas me payer un scooter » (alors que ses parents dressaient un tableau idyllique du périurbain), ou inversement dans un entretien d'un ménage ayant décidé de retourner en ville parce que la maman en a «marre de faire le taxi » lorsque l'un des enfants (sept ans) signifie clairement son désir de rester dans la fermette rénovée où il a «son atelier ». Dans ces deux cas, ressortent en fait les difficultés de certains membres de la famille face à une localisation résidentielle qui n'est que la résultante d'un consensus plus ou moins négocié en interne. Dans le périurbain lointain, le sentiment d'isolement des femmes qui cessent parfois de travailler en raison des difficultés de transport, a bien été montré par Rougé (2003). De même, ce sont les grands adolescents qui souffrent le plus de l'éloignement, la vie de leur groupe social se faisant en ville ou dans sa proche périphérie (multiplexes, etc.) ou bien se résume à « glander sous l'abribus » (une commune de notre échantillon a même construit un abribus pour les ados, situé au beau milieu d'une parcelle en friche...). Les contraintes externes et internes qui pèsent sur les individus incitent donc à articuler raisonnement au niveau de l'individu et raisonnement au niveau des ménages.

\section{Identités et territorialités}

Deuxièmement, la fréquentation plus ou moins quotidienne de l'espace environnant (la commune et de plus en plus un espace un peu plus large pour les activités de loisirs, la consommation quotidienne ou quelques services de base), ne traduit que très imparfaitement l'identité spatiale des ménages et des individus résidant dans le périurbain. Pour certains ménages ou individus, non seulement la ville est le lieu de travail, le lieu de consommation, le lieu de loisir et donc est prédominante en termes de pratiques spatiales et de temporalités, mais la ville est aussi le lieu de ces relations sociales pérennes et souvent plus impliquantes, moins formelles et plus profondes (les gens parlent d'amitié), construites anciennement avec les amis d'enfance, les amis des années lycée ou de l'Université, ceux avec qui on partage une 
passion commune, etc. Ce sont ces relations qui structurent profondément l'identité sociale de l'individu ou du ménage.

L'articulation entre identité spatiale et fréquentation des lieux nécessiterait donc un examen approfondi. Ce n'est pas parce que des ménages fréquentent les mêmes lieux qu'ils auront pour autant la même identité spatiale, cela dépend de la façon dont ils pratiquent les lieux. L'hypermarché de sortie de ville est un bon exemple : deux ménages résidant dans une même commune périurbaine ne vont pas le fréquenter selon les mêmes modalités. En particulier, ce sera une rupture dans le quotidien pour un ménage ne travaillant pas dans la grande ville, ce que ne ressentira pas le ménage travaillant en milieu urbain, alors même que les rythmes de fréquentation peuvent être identiques. Dans le premier cas, cela ne structure que faiblement l'identité spatiale, alors que dans le second, la position spatiale de l'hypermarché participe, avec bien d'autres repères, de la connaissance approfondie de l'espace urbain qui est constitutive d'une identité spatiale urbaine.

De plus, lorsque que certains lieux sont pratiqués de façon impliquée (exercice d'un pouvoir, attachement sentimental, etc.), une identité spatiale plus forte se construit. Certains lieux symboliques pas forcément pratiqués mais qui résonnent avec des formes d'identité profonde, contribuent également à forger l'identité spatiale des individus et des ménages. Il ne s'agit plus d'une territorialité viscérale, qui pouvait faire parler d'enracinés et de déracinés (Frémont et al. 1984), mais d'une territorialité partiellement choisie, en tous cas construite par le discours, mais aussi par les actes lorsque les individus s'impliquent dans la vie sociale locale. Cette territorialité est réversible en cas de déménagement, même s'il restera toujours une familiarité particulière avec cet espace.

D'autres facteurs génèrent un flou sur l'identité spatiale. Avoir un enfant qui fait ses études supérieures dans une grande ville même lointaine, génère un sentiment de familiarité, de connaissance avec cette ville, qui influe sur l'identité spatiale, alors que cette ville est peu ou pas fréquentée. Un néo-retraité, grand amateur de voyages itinérants en camping-car, parti plus de trois mois dans l'année, exprimait aussi, avec ses mots, le sentiment de ne plus avoir de véritable identité spatiale, sinon celle de «citoyen du monde » (et une identité sociale de « camping-cariste ») et en concevoir une grande liberté. Enfin, l'immédiateté des relations sociales par téléphone ou par Internet recompose parfois l'identité spatiale des individus, permettant une identité à distance, détaché de la simple fréquentation de lieux qui ne sont que des supports de la vie quotidienne. Le vrai sentiment identitaire est resté dans un lieu devenu mythique, fréquenté épisodiquement voir plus du tout, mais qui rejailli sur l'être social.

Ainsi, parmi les ménages de certaines communes du périurbain lointain, plusieurs identités spatiales coexistent. Certains individus vont avoir une identité spatiale réduite à la commune de résidence, avec un fort rapport au logement et une pratique plus ou moins impliquée de la commune de résidence, alors que l'éventuel lieu de travail urbain ne génère pas de sentiment d'appartenance. Dans la maison voisine, l'identité spatiale peut au contraire être à prédominance urbaine, complètement décentrée par rapport au logement, le lieu de résidence est donc moins signifiant, alors même que le rapport au logement peut être fort (mais il pourrait être fort ailleurs). Enfin, dans une troisième maison, l'identité spatiale peut être diffuse, associant le logement, une vague entité administrative (le département est souvent cité) et une référence à un espace lointain, dont on est originaire ou que l'on fréquente épisodiquement et qui fait office de territorialité rêvée.

\section{Apports pour le concept d'habiter :}

Ce travail empirique permet donc de proposer quelques apports pour la problématique de l'habiter. En fait, cela dépend du sens donné à l'habiter. Ce concept est actuellement de 
plus en plus utilisé dans les sciences sociales et, situé à la croisée de plusieurs disciplines, géographie, urbanisme, architecture, sociologie de l'habitat, anthropologie, avec des filiations désormais bien établies (Stock 2004), il est porteur d'un potentiel commun permettant de faire avancer la transdisciplinarité.

Il est toutefois utilisé diversement, avec plusieurs définitions pour le moins contrastées. Ces conceptions différentes sont en partie basées sur des échelles d'analyse différenciées.

Une première acception très courante en sociologie de l'habitat fait d'habiter le quasi synonyme de résider (Segaud, Brun et Driant 2003). Analysant les usages du logement, les modes d'habiter (Arbonville 1998), les pratiques de transformation du logement (et spécifiquement parfois de la maison individuelle), la mise en scène de la maison et de ses àcôtés (le jardin d'ornement ou le potager), le partage de l'espace entre membres du ménage, étudiant parfois les sociabilités liées au logement (Vervaecke 1987), ces travaux insistent sur le domicile. Dans ce cadre, nous pouvons au mieux apporter des éléments d'ordre factuels, et confirmer que dans le périurbain, la spatialité des familles est logiquement basée sur une pratique souvent intense et impliquante du logement. Mais cette approche centrée sur une seule échelle apparaît parfois peu géographique et doit être pondérée par l'importance très relative du logement pour certains ménages d'identité spatiale urbaine.

Une seconde acception, toujours centrée sur le logement, élargit le cercle de l'espace « habité » au quartier (Authier 2001), insistant beaucoup plus, au niveau thématique, sur les sociabilités avec les voisins, la fréquentation des commerces et des services de proximité, le rapport à l'école pour les ménages avec des enfants, la fréquentation des espaces verts ou de loisirs du quartier, ou encore la pratique sportive. Cette fois, les apports semblent plus importants, d'abord en infirmant en grande partie l'émergence d'un habiter périurbain spécifique dans les villes intermédiaires et moyennes. La présence dans le périurbain n'est parfois que passagère, s'intégrant dans un parcours résidentiel complexe, et les rapports à l'échelon local, commune périurbaine ou quartier, ne sont que peu différents de celui de familles résidant dans des lieux moins périphériques. Par ailleurs, l'éclatement des lieux de vie et l'aspect réticulaire des spatialités invitent également à ne pas raisonner à partir d'espaces emboîtés autour du logement, rendant en particulier obsolète l'approche en termes de coquilles entourant l'individu (Moles 1992), des espaces fort éloignés physiquement pouvant être proches dans l'esprit (Hoyaux 2003).

Enfin, une dernière acception élargit encore le champ d'action de l'habiter, incluant l'ensemble des pratiques spatiales sur la ville, voire celles liées au tourisme (MIT 2002) se déployant donc sur l'ensemble du monde, acception qui consiste peu ou prou à faire d'habiter un synonyme de spatialité ou d'espace vécu (Lévy et Lussault 2003). Cette approche, aux bases philosophiques plus affirmées, rend compte de la manière d'être au monde (Hoyaux 2002) et est celle qui se développe le plus à l'heure actuelle en géographie. Les résultats empiriques que nous avons présentés permettent de confirmer tout l'intérêt d'une approche par les pratiques spatiales (Stock 2004) en s'intéressant aussi à la façon dont les individus s'approprient ou non les lieux fréquentés, plaquant des comportements habituels sur un espace sans que celui-ci n'ait une résonance particulière pour l'individu ou, au contraire, avec la construction d'une familiarité donnant un sens fort à la pratique d'un lieu. Les lieux de référence de l'identité spatiale sont ainsi fortement éclatés et ne construisent pas obligatoirement une territorialité homogène, d'autant que malgré la proximité spatiale, sociale ou familiale, les individus construisent de plus en plus des référentiels différenciés.

Bibliographie:

Authier J.Y. (dir) (2001) : Du domicile à la ville, vivre en quartier ancien, Anthropos. 
Baudelle G., Darris G., Ollivro J., Pihan J. (2004) : Les conséquences d'un choix résidentiel périurbain sur la mobilité : pratiques et représentations des ménages, Cybergéo $\mathrm{N}^{\circ} 287,17 \mathrm{p}$.

Berger M. (1991) : L'urbanité des périurbains d'Ile-de-France : de la diversité à la ségrégation, Les Annales de la Recherche Urbaine N50, p56-63.

Bertrand J.R., Chevalier J., Dodier R., Gasnier A. (2000) : Le Mans. Peut-on changer la ville ? Anthropos.

Bonvalet C. et Dureau F. (2000), «Les modes d'habiter: des choix sous contraintes », in Métropoles en mouvement, Dureau F. et al (dir), Anthropos, p.131-153.

Brück L., Mairy N., Halleux J.M., Mérenne-Schoumaker B. (2001) : Les comportements résidentiels des ménages face à la problématique du développement durable, rapport SSTC, $16 \mathrm{p}$.

Di Méo G. (1998) : Géographie sociale et territoires, Nathan.

Dodier R. (2004) : L’espace dans les stratégies résidentielles des ménages, ESO N²1, p115123.

Dodier R. (2003) : Changements dans les flux domicile travail : de la ville industrielle à la «nouvelle » organisation urbaine, communication au colloque Rural - urbain : les nouvelles frontières. Permanences et changements des inégalités socio-spatiales, Poitiers 4- 6 juin 2003, à paraître aux PUR.

Estèbe, P. (2004) : Quel avenir pour les périphéries urbaines ?, Esprit $\mathrm{N}^{\circ} 303$.

Frémont A. (1976) : La région, espace vécu, Presses Universitaires de France, 2ème édition 1998.

Frémont A., Chevalier J., Hérin R., Renard J. (1984) : Géographie sociale, Masson.

Grésillon M. (1998) : La grande spatialité du vote d'extrême-droite en France, in Pumain D. et Mattéi M.F. (dir), Données urbaines II, Anthropos/Economica, p49-58.

Guilly C. et Noyé C. (2004) : Atlas des nouvelles fractures sociales en France, Autrement.

Haegel F. et Lévy J. (1997) : Urbanités, identité spatiale et représentation de la société, in Calenge C., Lussault M. et Pagand B. (dir), Figures de l'urbain ; des villes, des banlieues et de leurs représentations, Publications de la maison des sciences de la ville, p35-95.

Haegel F. (1998) : Une lecture spatiale des identités, in Haumont A. (dir), L'urbain dans tous ses états. Faire, vivre, dire la ville, L'Harmattan.

Haumont N. (1966) : les pavillonnaires, CRU.

Hoyaux A.F. (2002) : Entre construction territoriale et constitution ontologique de l'habitant : introduction épistémologique aux apports de la phénoménologie au concept d'habiter, Cybergéo $\mathrm{N}^{\circ} 102,18 \mathrm{p}$.

Hoyaux A.F. (2003) : Les constructions des mondes de l'habitant : éclairage pragmatique et herméneutique, Cybergéo $\mathrm{N}^{\circ} 232,23 \mathrm{p}$.

Jaillet M.C. (2004) : L'espace périurbain : un univers pour les classes moyennes, Esprit $\mathrm{N}^{\circ}$ 303, p40-60.

Knafou R. (dir) (1998), La société «nomade». Les mobilités géographiques d'aujourd'hui, Belin.

Lévy J. et Lussault M. (dir) (2003) : Dictionnaire de la géographie et de l'espace des sociétés, Belin.

Moles A. (1992): Vers une psycho-géographie, in Bailly A., Ferras, R., Pumain, D., Encyclopédie de géographie, Economica, p177-205.

Moles A. et Rohmer E. (1998) : Psychologies de l'espace, L'Harmattan.

Raymond H. (1998) : Habiter et vie quotidienne, in Logement et habitat, l'état des savoirs, La Découverte, p390-396.

Rougé L. (2003), Les nouvelles frontières du périurbain : accession à la propriété en maison individuelle et pratiques résidentielles des ménages modestes toulousains, communication au 
colloque Rural - Urbain : les nouvelles frontières: Permanences et changements des inégalités socio-spatiales, Poitiers 4- 6 juin 2003, à paraître aux PUR.

Sajoux P. (2004) : Habiter en espace périurbain et usages de la voiture, ESO N²1, p125-134.

Segaud M., Brun J. et Driant J.C. (dir) (2003) : Dictionnaire de l'habitat et du logement, Armand Colin.

Stock M. (2004) : L'habiter comme pratique des lieux géographiques, EspacesTemps.net, http://espacestemps.net/document1138.html 